\title{
Uso y apropiación de las TIC en educación
}

\author{
Oscar Yecid Aparicio Gómez ${ }^{1}$
}

Recibido: 12-07-2018

Aceptado: 17-09-2018

\section{Resumen}

El presente artículo $^{2}$ pretende describir y analizar los usos de las TIC en una Institución Educativa del Municipio de Chía, y la comprensión del uso de las TIC como herramientas cognitivas, así como proponer mejoras dirigidas a la integración de las TIC en la docencia de profesores y a la impregnación curricular de las TAC. Esta investigación recoge las impresiones generales y particulares de una comunidad educativa a través de la interpretación de los datos recogidos por medio del Instrumento seleccionado con respecto a cómo se desarrollaron procesos de Investigación usando las TIC, y procurando identificar particularmente cómo se usaron las TIC para la investigación en cada uno de los Grupos de Investigación de una Institución Educativa del Municipio de Chía (Colombia).

Palabras clave:transferencia del conocimiento, TIC, instituciones educativas, colegio, investigación, metodología de la investigación, instrumentos de investigación, pedagogía.

1. Doctor en Filosofía (2006) y Doctor en Educación (2015) por la Universidad de Barcelona. Profesor y Editor.

Bogotá - Colombia.

Correo electrónico: oscar.yecid@gmail.com

ORCID: 0000-0003-3535-6288

2. La información puede ampliarse en: Aparicio, Ó. Y. (2015). Las TIC como herramienta cognitiva para la investigación escolar (Tesis doctoral, Universitat de Barcelona, Barcelona, España). Recuperado de http://hdl.handle.net/10803/369830 


\title{
Use an appropiationof ICT in education
}

\begin{abstract}
This article aims to describe and analyze the uses of TIC in an Educational Institution of the Municipality of Chia, and the understanding of the use of TIC as cognitive tools, as well as propose improvements aimed at the integration of TIC in the teaching of teachers and the curricular impregnation of the TAC. This research gathers the general and particular impressions of an educational community through the interpretation of the data collected through the selected Instrument with respect to how research processes were developed using TIC, and trying to identify particularly how TIC was used for the research in each of the Research Groups of an Educational Institution of the Municipality of Chia (Colombia).
\end{abstract}

Keywords: knowledge transfer, ICT, educational institutions, school, research, research methodology, research instruments, pedagogy.

\section{Introducción}

El objetivo general del cuestionario es conocer el estado final de los Proyectos de Investigación en una Institución Educativa del Municipio de Chía.

Sobre lo que se quiere conocer acerca del estado final de las investigaciones, se encuentran: una breve recopilación del proyecto, el instrumento que se usó en la investigación (los objetivos, una breve descripción y la manera en la que se construyó y se distribuyó, la muestra), los resultados obtenidos (si se tuvo que realizar un proceso de preparación y análisis, las respuestas que obtuvieron, las preguntas que les parecieron más importantes para el análisis y los resultados del instrumento), la interpretación que se dio a los resultados obtenidos, el uso que le dieron a las TIC durante la realización del proyecto y las conclusiones y dificultades del Proyecto de Investigación. 


\section{Metodología}

El cuestionario se compone de cinco (5) ítems y sus correspondientes subítems (13) que emergen como resultado de las preguntas orientadoras, practicadas en el cuestionario, y que se pueden ver a continuación de cada uno de ellos. Los ítems irán precedidos por el número de Instrumento y su número de orden correspondiente.

A continuación, se presentan preguntas diseñadas mediante ítems y subítems con el fin de conocer los documentos finales de investigación que nos permiten conocer los procesos que se han llevado a cabo por una Institución Educativa del Municipio de Chía.

\section{Instrumentos utilizados en los proyectos de investiga- ción}

111 ¿Cuáles son los objetivos del cuestionario? Objetivo: Exponer los objetivos previstos para la realización del cuestionario.

112 ¿Cómo describe la estructura del cuestionario? Objetivo: Conocer la estructura que presenta el cuestionario.

113 ¿Cuál fue el proceso de construcción y distribución del cuestionario? Objetivo: Distinguir el proceso seguido para la construcción del cuestionario, así como su distribución.

\section{Selección de la muestra}

121 ¿Cuál es la población a la que va dirigido el cuestionario? Objetivo: Identificar la población a la que potencialmente se esperaría aplicar el cuestionario que se construyó.

122 ¿Cuál fue la muestra participante? Objetivo: Conocer a quienes efectivamente se les aplicará el cuestionario construido.

\section{Resultados}

I31 ¿Cuál fue la preparación y el análisis previo que tuvieron que realizar? Objetivo: Determinar cómo se hizo el análisis previo y la preparación para la aplicación del Instrumento. 
132 ¿Cuáles fueron las preguntas que orientaron el análisis? Objetivo: Establecer cuáles fueron las preguntas que se tuvieron en cuenta para realizar el análisis de la información recolectada en el cuestionario.

133 ¿Cuáles fueron las respuestas que se obtuvieron? Objetivo: Conocer las respuestas que se obtuvieron del cuestionario.

I34 ¿Cuáles fueron los resultados del cuestionario? Objetivo: Reconocer cuáles son las respuestas que se obtuvieron con la realización del cuestionario.

I35 ¿Qué interpretación ha dado a estos resultados? Objetivo: Determinar cuál es la interpretación que dio a la información recogida en el cuestionario.

\section{5/I4. Uso de las TIC para la investigación}

141 ¿Cuál fue el uso que se dio a las TIC durante el desarrollo del Proyecto de Investigación? Objetivo: Evaluar el uso que se dio a las TIC en la realización del Proyecto de Investigación para conocer las herramientas que se usaron durante el proceso.

\section{5/15. Conclusiones y limitaciones}

151 ¿Cuáles fueron las conclusiones de la investigación? Objetivo: Establecer las conclusiones a las que llegó el Grupo de Investigación con la realización del proyecto.

152 ¿Cuáles fueron las limitaciones de la investigación? Objetivo: Identificar las limitaciones que se presentaron en los Grupos de Investigación durante la realización de los Proyectos de Investigación.

\section{Resultados}

Cuando se realice una citación textual sobre una respuesta de algún relator se enuncia con el prefijo $05 / \mathrm{R}^{3}$ seguido por un número de acuerdo

3. El 05 hace referencia al quinto instrumento que se ha aplicado y "R" corresponde al número asignado al relator. 
con el grupo que corresponda. A continuación, se presenta el análisis de los resultados del cuestionario procedente de los diversos Grupos de Investigación.

\section{5/R1. Descubro lo maravilloso que es mi cuerpo}

05/10. Introducción. Descubrir el cuerpo y lo esencial para la vida como características para comprender la evolución de la ciencia (de la calle, Malaver, Gallego, Rodríguez, Flórez, Saldaña, 2014) y de la conexión con el día a día, lo que somos, sentimos, anhelamos, soñamos, hacemos y expresamos.

05/I1. Instrumento. El objetivo de la encuesta es el diagnóstico de la población en cuanto al conocimiento del cuerpo, para lo cual se decidió usar un cuestionario. Para construir el cuestionario, se usaron preguntas basadas en los temas que fueron vistos durante el proyecto y que fueron relevantes para los estudiantes. El resultado fueron cinco preguntas para conocer lo que opinan los estudiantes sobre la importancia de algunas de sus partes del cuerpo, los implementos necesarios para el uso diario y su relación con el cuidado del cuerpo y la función del sistema digestivo. Para la distribución se envió un correo electrónico a los padres de familia de grado Preescolar para que los estudiantes realizaran el cuestionario en compañía de los padres.

05/I2. Selección de la muestra. La población a la que va dirigida el cuestionario son estudiantes de jardín y transición del Colegio. Como la población no es muy grande, se tomó toda la población como muestra.

05/13. Resultados. Mediante Google Drive se recolectan los datos, como son encuestas con respuestas cerradas no se hace un análisis previo de las respuestas obtenidas en el cuestionario. Lo que más resaltó la profesora de este curso fue la motivación para contestar el cuestionario, esto de acuerdo con las opiniones de lo que se ha vivido en el Colegio y de lo que han vivido. Se obtuvieron 23 respuestas, que corresponde al número de familias a quienes se dirigió el cuestionario. Para el Grupo de Investigación las preguntas más importantes fueron: ¿qué parte del cuerpo te parece más importante?, ¿para qué sirve el cepillado de los dientes?, ¿qué implementos de aseo usas para la higiene de tu cuerpo al bañarte?, ¿cuál es la función del sistema digestivo?, y, ¿de qué están encargados 
los pulmones? Los resultados que arrojaron las encuestas fueron los siguientes: La parte del cuerpo más importante para los encuestados son los órganos de los sentidos (48\%), seguido por la cabeza (48\%).

- Todos los encuestados opinan que el cepillado de los dientes sirve para mantenerlos limpios y sanos.

- Todos los encuestados opinan que los implementos de aseo al bañarse el cuerpo son agua, jabón y champú.

- Para los encuestados el sistema digestivo está encargado de digerir los alimentos.

- Según los encuestados ninguna de las respuestas que estaban en las opciones es la que satisface la función de los pulmones.

La interpretación se realizó mediante la socialización con los estudiantes. A través del cuestionario afianzaron los conocimientos y alcanzaron los objetivos.

05/14. Uso de las TIC para la investigación. La profesora resalta la importancia de las TIC en el proceso investigativo, así como el uso que dieron a las TIC durante el Proyecto de Investigación:

- "Actividades Complementarias" en la Plataforma del Colegio.

- Exposiciones de los estudiantes para dar a conocer los temas a sus compañeros.

- Video creado por las tutoras de Preescolar con ayuda

de estudiantes. Con esto se da a conocer el proyecto a

los compañeros de Educación Básica Primaria y Educación Secundaria y Media Académica

- Fotos del trabajo realizado en las clases.

- Google Drive para la construcción del cuestionario.

- Correo electrónico para la distribución del enlace del cuestionario.

05/15. Conclusiones y limitaciones. Entusiasmo y motivación por parte de los estudiantes en el proyecto, puede verse en las actividades que se realizaron a lo largo del proyecto. Además, aportaban en el desarrollo con sus opiniones personales en relación con los temas tratados. Se logró afianzar valores de respeto y solidaridad (Pérez, 2013), además se descubrieron talentos en los estudiantes relacionados con lo que les agrada. La mayor limitación fue el tiempo para la realización de las actividades que 
se realizaron. Además, no se tienen los recursos suficientes y la calidad de la conexión a Internet no es muy buena.

\section{5/R2. El juego, un camino hacia las matemáticas}

05/10. Introducción. La investigación se basa en el área de matemáticas como un gran dolor de cabeza para los estudiantes, perdiendo el hecho de que es un área del conocimiento que hace parte del lenguaje de la naturaleza. Por lo que se deben buscar nuevas metodologías para el aprendizaje de esta importante área del conocimiento. En el Proyecto de Investigación se busca por medio del juego enseñar conocimientos matemáticos, y conocer las ventajas que el juego logra desarrollar en los niños en las matemáticas.

05/I1. Instrumento. El principal objetivo del cuestionario era identificar las causas que producen la fobia a las matemáticas, relación juego-matemáticas, conocer opinión sobre la metodología usada en matemáticas, para lo cual realizaron una encuesta. El cuestionario contiene 10 preguntas con múltiples opciones y de la cual solo se puede seleccionar una respuesta. En las preguntas que se realizaba a la población se buscaba conocer la relación de los estudiantes con la clase de matemáticas o las matemáticas en general (operación que más se le facilita, cómo se sienten con la metodología de la clase, utilidad de las matemáticas, entre otras) y la relación entre los estudiantes y los juegos (motivación para desarrollar un juego, el tipo de juegos que más les gusta, entre otros). Se diseñó con ayuda de los estudiantes del Grupo de Investigación, se realizó la discusión y análisis de los objetivos para lograr la construcción de las preguntas por medio de socialización. Luego, se publicaron las preguntas en una encuesta en Google Drive. Se requirieron dos sesiones para la realización de las preguntas. Se distribuyó el enlace para realizar el cuestionario en la Plataforma Virtual del Colegio, el cual estuvo disponible durante 8 días.

05/12. Selección de la muestra. Estudiantes de Educación Básica Primaria de la Institución educativa. Ya que la población no es muy grande, se aplicó el cuestionario a todos los estudiantes de Educación Básica Primaria, con un total de 74 participantes.

05/13. Resultados. Se obtuvieron 40 respuestas de estudiantes de los grados de Educación Básica Primaria, de un total de 74 estudiantes encues- 
tados. Se realizó la recopilación de los resultados del cuestionario y se pusieron en Excel para realizar el análisis por medio de gráficos y tablas. Las preguntas que resaltó el profesor líder de la investigación fueron: ¿tienes fobia a las matemáticas?, ¿qué actividades se pueden realizar en clase de matemáticas para mejorar el aprendizaje?, ¿Crees que por medio de los juegos se puede aprender matemáticas?, ¿cuál de los siguientes métodos te parece mejor para evitar la fobia a las matemáticas?, y, ¿te gustaría crear algún juego matemático? Los resultados que arrojó el instrumento fueron:

- La operación que más se les facilita es la suma (65\%), luego está la multiplicación (20\%).

- 90\% de los estudiantes dijeron no tener fobia a las matemáticas.

- $93 \%$ creen que es posible aprender matemáticas por medio de un juego.

- $38 \%$ de los estudiantes prefieren los juegos deportivos, seguido por un 33\% que prefieren los juegos de computador o en línea.

- $43 \%$ opinan que se pueden realizar ejercicios sobre los temas vistos para mejorar el aprendizaje de las matemáticas, el $30 \%$ opinan que se puede mejorar por medio de juegos.

- $33 \%$ opinan que las matemáticas se pueden aprender desde los 5 años, 30\% opinan que desde los 3 años y 23\% opinan que desde el primer año.

- 33\% de los estudiantes opinan que el mejor método para evitar la fobia a las matemáticas son los juegos. Mientras que el $25 \%$ opinan que el mejor método es prestar atención a los profesores y otro $25 \%$ opina que lo mejor es hablar con el profesor sobre los problemas en la materia.

- 60\% de los estudiantes opinan que las matemáticas sirven para todo lo que hacen en su vida diaria. Un 40\% está dividido entre que sirve para aprender sobre números y sus operaciones y para resolver problemas.

Se presenta la interpretación que el Grupo dio a estos resultados:

- "En general los estudiantes de Educación Básica Primaria de la Institución Educativa del no sienten que el área de matemáticas les transmita alguna fobia". 
- Los estudiantes "sienten que el juego es un método que les permite aprender de una manera práctica y divertida dejando de lado las dificultades y realizando un trabajo colaborativo con sus compañeros".

- Los estudiantes "muestran preferencia por los juegos informáticos" y es una oportunidad para que los profesores mejoren sus metodologías aprovechando estas preferencias (Konieczny, 2015).

05/14. Uso de las TIC para la investigación

- Para la recolección de información: YouTube, Internet.

- Diseño y divulgación de las encuestas - Google Drive

- Recolección y organización de información en Excel.

- Aplicación Hopscotch ${ }^{4}$ para el desarrollo de juegos matemáticos - basada en juego rayuela (Ayuda a iniciar a los niños en la programación. Bloques de colores y cada uno tiene una función o comando para ejecutar. Permite definir personajes con nociones básicas geométricas y matemáticas).

- Plataforma Virtual del Colegio para distribución de los cuestionarios.

05/15. Conclusiones y limitaciones

- Se identificó que en el Colegio muy pocos son los estudiantes tienen fobia por las matemáticas.

- Las principales causas de bajos resultados y miedo son la metodología utilizada y la dedicación a indagar sobre lo que se les dificulta.

- El juego es un buen camino para la enseñanza, ya que se divierten y recuerdan más fácilmente por lo vivencial.

- Las matemáticas forman parte de la vida cotidiana y permiten alcanzar múltiples objetivos como que niños de Educación Básica Primaria programen un juego.

- El aprendizaje conceptual complementado con juegos, ayudan a obtener mejores resultados.

\section{5/R3. Las matemáticas y la vida: una relación imperceptible}

05/10. Introducción. Se presentan dificultades en el aprendizaje matemático en los estudiantes, es necesario buscar ayudar a los estudiantes para 4. Véase: https://www.gethopscotch.com/ 
mejorar en el aprendizaje de las matemáticas buscando las causas y consecuencias de esta dificultad de aprendizaje. Para el Grupo de Investigación es necesario publicar los resultados para demostrar que las matemáticas se usan en todo momento. En cada etapa de la investigación se iba construyendo un adelanto del producto final.

05/I1. Instrumento. Los objetivos de la encuesta fueron: indagar sobre la metodología, motivación y adquisición del conocimiento matemático, investigar sobre las metodologías y recursos empleados en la enseñanza de las matemáticas, conocer los gustos matemáticos de los estudiantes en su vida escolar y consultar sobe los tipos de didáctica que se deben aplicar para un mejor aprendizaje matemático de los futuros estudiantes. Para lo cual se decidió usar un cuestionario.

El cuestionario resultante fue una encuesta con 16 preguntas, divididas de la siguiente manera: 4 preguntas cerradas para conocer la forma de aprendizaje que tuvieron los encuestados, 6 preguntas cerradas con el objetivo de conocer la rama de las matemáticas que más les Ilamaba la atención y la forma con la cual fueron motivados para que les guste, y finalmente, 6 preguntas abiertas, que buscan recolectar la opinión de los encuestados sobre cuáles son las mejores didácticas para aprender matemáticas. Para la construcción del cuestionario se realizaron dos encuentros con los integrantes del Grupo de Investigación, en estos se diseñaron, discutieron y aprobaron las preguntas. Se construyó el cuestionario en Google Drive. Se distribuyó el enlace del cuestionario en la Plataforma Virtual del Colegio.

05/I2. Selección de la muestra. Estudiantes de los grados Sexto, Séptimo, Octavo, Noveno y Décimo. Ya que la población no era muy grande, se decidió realizar el cuestionario sobre toda la población, de estos grados participaron 40 estudiantes.

05/I3. Resultados. Se obtuvieron respuestas de 26 estudiantes, escogidos aleatoriamente Educación Secundaria y Media Académica. Se trasladaron los resultados desde Google Drive a Excel para su posterior análisis. Con respecto a las preguntas abiertas, se realizó la socialización de las respuestas para poder analizarlas en grupo. Según el titular de la investigación, las preguntas más importantes fueron las siguientes:

- Preguntas sobre la manera de aprender matemáticas: ¿de qué manera aprendiste matemáticas? ¿Cómo te enseñaron matemáticas en tu Colegio? 
- Gustos en las matemáticas: de los temas que has visto ¿hay alguno que te haya llamado la atención? ¿Qué clase o rama de las matemáticas es la que más te gusta?

- Motivación para el aprendizaje: ¿de qué manera se aprendería mejor matemáticas? ¿De qué manera crees que se pueda motivar a un estudiante para aprender matemáticas?

Los resultados que arrojaron las encuestas fueron los siguientes:

- La mayor parte de los estudiantes manifestaron aprender matemáticas virtualmente, repitiendo, jugando, contando con los dedos, con ejercicios y practicando (opción todas las anteriores).

- 54\% de los estudiantes opinan que sí les ha llamado la atención alguno de los temas matemáticos, solo un $8 \%$ opinan que no.

- 46\% de los estudiantes manifestaron que la mamá fue la que lo apoyó cuando aprendió matemáticas, mientras que el $31 \%$ dijeron que ninguno de los papás los ayudó.

- $62 \%$ de los estudiantes dicen que su enseñanza de matemáticas se hizo con muchos ejercicios y solo el 19\% manifiestan que su enseñanza se hizo jugando.

- La geometría es el área de las matemáticas que más les gusta (35\%), al 23\% lo que más les gusta es la aritmética, al $19 \%$ lo que más les gusta es la estadística, mientras que al 23\% no les gusta ninguna de las ramas de las matemáticas.

- $73 \%$ de los estudiantes manifiestan que se aprendería mejor matemática de una manera más didáctica antes que de la manera tradicional.

- La mayoría de los estudiantes (69\%) no conocen ningún juego para aprender matemáticas.

- $58 \%$ de los estudiantes opinan que se puede motivar a los estudiantes para aprender matemáticas por medio de métodos didácticos, mientras que un $54 \%$ de los estudiantes opinan que se puede generar esa motivación utilizando la tecnología como una fuente de aprendizaje.

- 58\% de los estudiantes opinan que el profesor de matemáticas los motiva para aprender en esta área.

- $50 \%$ de los estudiantes opina que ni el profesor, ni los cálculos los motiva en el aprendizaje de las matemáticas. 
- $46 \%$ de los estudiantes opinan que se puede aprender matemática didácticamente por medio de juegos y el $42 \%$ opinan que se puede aprender didácticamente mediante la solución de situaciones problema de la vida cotidiana.

- La mayoría (65\%) de los estudiantes aprendieron matemáticas por medio del método tradicional.

La interpretación que le dieron a los resultados por parte del Grupo de Investigación fue que "a pesar de que la mayor parte de los estudiantes opinan que se puede motivar a aprender por medio de métodos didácticos, no han tenido una relación estrecha con este sistema, ya que la mayoría no conoce ningún juego para aprender matemáticas"

05/14. Uso de las TIC para la investigación. El uso que le dieron en este Grupo de Investigación a las TIC durante el desarrollo de la investigación fue:

- Google Drive para la indagación y recolección de información (diseño de encuestas)

- Hojas de Excel para el análisis de los datos.

- Scratch como herramienta para crear juegos matemáticos en uno de los campos de interés para los estudiantes y despertar curiosidad en el estudiante

05/15. Conclusiones y limitaciones. La mayor parte de los encuestados aprendió matemáticas de la manera tradicional. Uno de los factores influyentes sobre los estudiantes son los profesores, la población se siente motivada a aprender por parte del profesor. A pesar de que la mayor parte de los estudiantes opinan que se puede motivar a aprender por medio de métodos didácticos, no han tenido una relación estrecha con este sistema, ya que la mayoría de estudiantes no conoce ningún juego para aprender matemáticas. Con respecto a las limitaciones, se destaca, según el profesor, que había un desconocimiento de los días en los que se harán las sesiones de los Grupos de Investigación. Igualmente, no hay suficiente participación por parte de los estudiantes para aportar ideas y ser autodidactas, no entregan los compromisos en el tiempo de entrega estipulado. Finalmente, no se pudieron realizar con la suficiente frecuencia las asesorías estudiante-profesor para la corrección de avances en los compromisos que se han propuesto. 


\section{5/R4. Creando conciencia, animales en vía de extinción}

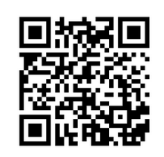

05/I0. Introducción. El proyecto está enfocado en la conciencia hacia la protección de los animales en vía de extinción, para lo cual se ha realizado una serie de consultas. Los estudiantes del grupo presentan un conocimiento previo frente al contexto del animal elegido lo que se tomó como base para la construcción de las preguntas, y luego se usó como diagnóstico para continuar con las siguientes fases mediante el uso y la aplicación de herramientas TIC. Durante el tiempo de investigación se participará de la observación de campañas ambientales y del cuidado silvestre, y se accederá a trabajos realizados en la web sobre el tema. Cada estudiante inició la creación de un texto literario o informativo, de acuerdo con su consulta realizada, que evidencian las causas y consecuencias que acaban con una especie silvestre. Se realizó la publicación de estos textos con público los estudiantes del Colegio, para finalizar con una encuesta aplicada a los estudiantes para dar respuesta a lo que se buscaba con el proyecto.

05/I1. Instrumento. Los objetivos del cuestionario que realizó este Grupo de Investigación fueron: diagnóstico sobre el reconocimiento de las posibles causas y consecuencias relacionadas con la extinción de algunas especies animales silvestres, conocer la opinión de los estudiantes sobre las campañas de protección animal en vía de extinción, conocer las soluciones por las que más se inclinan los estudiantes frente a la extinción animal e identificar el impacto del medio por el que se divulga la información sobre el tema. Para ello se plantearon 6 preguntas con opción múltiple con respuesta única. Para la construcción del cuestionario, se basó en la elaboración de cuentos y textos informativos de acuerdo con las consultas realizadas por los estudiantes, además los estudiantes observaban algunos videos relacionados sobre el tema. De esta manera, a los estudiantes les surgían preguntas que compondrían el cuestionario final. Se construyó el cuestionario en Google Drive para que sea atractivo. Igualmente, se distribuyó el video y el enlace de la pregunta en la Plataforma Virtual del Colegio para los estudiantes de Educación Básica Primaria.

05/12. Selección de la muestra. La muestra participante eran los estudiantes de Educación Básica Primaria de una Institución Educativa del Municipio de Chía, en principio, el cuestionario se pensaba aplicar a todos los 
estudiantes. Pero solo 15 de 74 estudiantes de Educación Básica Primaria respondieron el cuestionario.

05/I3. Resultados. Se obtuvieron 15 respuestas por cada pregunta planteada. Para preparar el terreno para el análisis, se comentaron los resultados esperados del cuestionario. Google Drive permite un fácil acceso a la edición del cuestionario, tiene estructura de vista previa y las respuestas son entregadas en tablas y gráficos a modo de resumen.

Para el Grupo de Investigación, las preguntas más relevantes son: después de ver el video, ¿qué tanto es ahora consciente del daño causado por la sociedad al animal nombrado en el video?, ¿qué tanto este medio interactivo y las herramientas TIC utilizadas en él, permiten la divulgación de información sobre el tema?, ¿cree que esta forma de divulgación de la información ayuda a generar conciencia en las personas sobre la temática de extinción silvestre? Los resultados que arrojaron las encuestas distribuidas entre los estudiantes fueron:

- Después de ver el video 100\% se sienten más conscientes en el daño sobre el animal.

- Después de ver el video 100\% reconoció la información de los peligros que afectan a los animales y los llevan a su extinción.

- 53\% identificó la caza ilegal como la causa de extinción en el video, el $27 \%$ identificó la contaminación y el $20 \%$ la deforestación.

- 73\% opinan que el video y las herramientas TIC en la Plataforma permiten en gran medida la divulgación sobre la extinción de los animales, mientras que un $27 \%$ opinan que sirve poco.

- 93\% opinan que los videos y la distribución por canales interactivos sirven para generar conciencia sobre la extinción silvestre.

- Con respecto a la pregunta sobre cuál solución les agrada más y en la cual están dispuestos a contribuir ellos. El 33\% manifestaron que sería la protección de la flora natural, otro $33 \%$ ayudarían con la reserva animal, un $13 \%$ con campañas de divulgación en medios de comunicación, otro $13 \%$ por medio del reciclaje y un $7 \%$ opinan que en menos consumo de material contaminante. 
En cuanto a la interpretación que dieron de estos resultados, se puede resaltar lo siguiente:

- Se puede evidenciar que los estudiantes que vieron en su totalidad se sienten más conscientes en cuanto al daño que ha causado la sociedad sobre el animal del video, lo que indica que el material consultado ayudó en el resultado. Además, el medio por el que se intentó realizar la concientización sirve para generar conciencia.

- Todos los estudiantes sienten haber captado la información sobre los peligros que afectan a los animales y llevan a la extinción silvestre. Esto hizo que lograran reconocer algunas de las causas de esta extinción, como lo demuestra una pregunta en el cuestionario.

- La mayor parte de los estudiantes sienten que el uso de herramientas TIC y un medio como la Plataforma tecnológica es una buena manera de concientizar sobre la extinción de animales.

- También se pudo demostrar con esta investigación que los estudiantes desean contribuir con el cuidado de la fauna silvestre en vía de extinción, de alguna manera.

\section{5/I4. Uso de las TIC para la investigación}

- Medio para recoger y presentar la información.

- Consulta de información en Internet.

- Materializar el resultado del análisis investigativo por medio de un video publicado en Plataforma Virtual.

- Google Drive para encuestas.

\section{5/15. Conclusiones y limitaciones}

- Las personas desean contribuir con el cuidado de la fauna silvestre en vía de extinción, de alguna manera.

- Se logró crear conciencia en los estudiantes del Colegio sobre los animales en vía de extinción.

- Los recursos interactivos y herramientas TIC pueden servir como medio para la conservación del medioambiente y de los seres vivos.

- El Grupo de Investigación logró ahondar en los temas y aprender más sobre el uso de herramientas TIC en la educación (Aparicio, 2018). 
- Entre las limitaciones se destaca que los espacios o salas interactivas no siempre estaban disponibles. Igualmente, el poco tiempo no permitía un control más riguroso o un mayor acompañamiento del proceso y el desarrollo.

\section{5/R5. Literatura en vía de extinción}

05/I0. Introducción. El proyecto está basado en la importancia de la literatura en la formación personal de los estudiantes y la necesidad de profundizar y ampliar los conocimientos desde la lectura consciente teniendo como base los movimientos literarios y las obras más representativas a lo largo de la historia (de Almeida, Santos, Porto, 2017). Basándose en que la literatura tiene un papel importante en la maduración para los adolescentes y tiene una relación directa con otras ramas del conocimiento, de manera que pueden ampliar la visión de la realidad. Por lo tanto, se tiene en cuenta para el desarrollo de investigación la literatura en vía de extinción para que los estudiantes valoren la calidad de los textos que leen, teniendo como referencia un clásico de la literatura como lo es el libro "el extraño caso del Dr. Jekyll y Mr. Hide" (libro base de la investigación).

05/17. Instrumento. Los objetivos del cuestionario son: conocer lo que opinan los estudiantes sobre la literatura, conocer quienes determinan una concepción de literatura, identificar las razones que llevan a comprar un libro, conocer la valoración de los encuestados al contexto de los autores y conocer lo que opinan los estudiantes con respecto a la literatura actual. Además, en relación con la idea o concepción de literatura se quiere conocer los diferentes elementos a la hora de comprar un libro, vida del autor, contextos de la obra y factor económico. Por lo tanto, se decidió usar una encuesta.

El cuestionario resultante, realizado con Google Drive. consta de 16 preguntas, 7 de opción múltiple con única respuesta y 9 preguntas abiertas. La construcción del cuestionario se hizo con ayuda de los estudiantes. Con ayuda de las conversaciones de la lectura del texto base (clásico de la literatura). Se redactaron las preguntas abiertas teniendo en mente la interpretación de las ideas de los estudiantes frente al tema, mientras que se tenían unas cerradas que buscaban conocer la opinión puntual. 
05/I2. Selección de la muestra. Los estudiantes de Educación Básica y Media Académica de una Institución Educativa del Municipio de Chía fueron seleccionados como muestra, ya que el número no era muy alto. A pesar de esto, los resultados variaron de acuerdo con la participación voluntaria de los estudiantes.

05/I3. Resultados. De 183 estudiantes, a quienes se les envió la encuesta, solo 35 estudiantes respondieron a las 16 respuestas planteadas. Para la preparación y el análisis previo se tuvieron algunas conversaciones sobre el libro base (clásico de la literatura) para lograr interpretar las respuestas de los estudiantes. Además, se tuvo un plan de trabajo para la conceptualización y creación de un texto por parte del equipo de investigación.

Las preguntas más importantes para el Grupo de Investigación fueron: ¿cuáles son los estándares que se deben cumplir para que una producción textual sea categorizada como literatura?, ¿cómo podemos diferenciar un texto literario de otro que no lo es?, y, ¿quién determina qué es o no es la literatura? Los resultados que arrojaron las encuestas, en sus respuestas más significativas, fueron los siguientes:

- $63 \%$ no sabe quién determina qué es y qué no es la literatura, mientras el resto saben que existen entes para determinar qué es literatura como expertos, corrientes y críticos de literatura.

- $83 \%$ de los estudiantes estaban leyendo un libro en el momento del cuestionario.

- $35 \%$ dice que decide leer un libro por gusto personal, $21 \%$ dicen que por curiosidad y $15 \%$ por recomendación. Mientras que por imposición solo un $3 \%$.

- $23 \%$ dicen que a la hora de comprar un libro lo hacen por la temática, $14 \%$ dicen que, por el autor, $14 \%$ extensión y $13 \%$ por el índice, entre otras respuestas.

- $85 \%$ opinan que es importante conocer la vida y obra del autor en un libro.

- $29 \%$ opinan que la fama es el motivo por el cual las personas que no se dedican a la literatura deciden escribir libros.

Se pudo determinar que el abandono de un libro que se comienza a leer ocurre por gusto personal, curiosidad, falta de intriga y/o el tiempo de la lectura. 
05/14. Uso de las TIC para la investigación

- Recolección de datos.

- Difusión y presentación final de datos.

- Recolección y jerarquización de información.

- Creación y difusión del cuestionario (Plataforma del Colegio).

- Generadores de mapas conceptuales.

- Elaboración de encuestas.

- Herramientas de búsqueda de información.

- Presentaciones para mostrar el proceso investigativo.

- Examtime para la elaboración de presentación de mapas conceptuales.

- keynote: generador de presentaciones para iPad.

- Issu: generador de revistas, para presentar las reseñas literarias.

- Correo electrónico: con el objetivo de mantener comunicación con los estudiantes. Avances y productos del proyecto.

- Glogster: para la realización de carteleras.

05/15. Conclusiones y limitaciones. Se logró identificar los criterios establecidos que pueden llegar a enmarcar una producción como literatura. El determinante de qué es o no literatura, se basa en los gustos personales y de los conocimientos que se tienen. Además, se examinó a fondo un clásico de la literatura. Se lograron tres tipos de producciones textuales: ensayos, artículos de opinión y reseñas literarias. Se elaboró un decálogo del buen lector. La mayor limitación es el tiempo, no lograban desarrollar todas las actividades programadas en las sesiones. No todos los estudiantes realizaron la lectura del libro base, además existe la limitación en la producción y en los aportes a la investigación, en general (3 de 20).

\section{5/R6. Cultivos hidropónicos y agricultura sostenible}

05/IO. Introducción. Desde el comienzo de la humanidad se establecieron cultivos para suministrar alimentos a las personas. Con base en esto, los cultivos hidropónicos ofrecen una amplia comodidad ya que no necesitan grandes terrenos para generar sus frutos, simplemente los nutrientes. También se pueden obtener plantas ornamentales o pasto hidropónico para recreación. Esto contribuye a optimizar el medio donde se plantan ya que satisfacen las necesidades alimenticias de los seres humanos. Por 
lo tanto, la agricultura sostenible es la cual satisface las necesidades básicas de alimentos de una forma económicamente viable.

05/I1. Instrumento. El objetivo del cuestionario es conocer los niveles teóricos de los estudiantes de acuerdo con la temática de los cultivos hidropónicos, además de conocer la opinión de los estudiantes en cuanto a esta temática. Para esto, se decidió hacer una encuesta. El cuestionario resultante consta de 8 preguntas de selección múltiple con única respuesta. Dos de esas preguntas tenían un campo para elaborar mejor la respuesta, ya que al responder una de las opciones se habilitaba un campo de texto. En cuanto a la construcción del cuestionario, las preguntas fueron elaboradas por el Grupo de Investigación con la información recopilada en las fases previas a la construcción del cuestionario.

05/12. Selección de la muestra. La muestra participante del cuestionario son los estudiantes de Educación Básica Primaria de una Institución Educativa del Municipio de Chía. La muestra fue de 30 estudiantes de Educación Básica Primaria, escogidos aleatoriamente entre los grados Tercero, Cuarto y Quinto.

05/I3. Resultados. Treinta (30) estudiantes de Educación Básica Primaria respondieron a las 8 preguntas. Como un medio de preparación y análisis previo se construyó una matriz cualitativa para realizar el análisis de las respuestas de los estudiantes. Además, se compartieron experiencias personales en el Grupo de Investigación. Los resultados que arrojaron las encuestas realizadas fueron:

- 67\% de los estudiantes opinan que los cultivos hidropónicos crecen más rápido que los de la tierra.

- $90 \%$ de los estudiantes opinan que en Colombia se realizan cultivos hidropónicos.

- $87 \%$ de los estudiantes opinan que los cultivos hidropónicos ayudan a la economía colombiana.

-73\% de los estudiantes opinan que el compostaje es descomposición de la materia orgánica.

-47\% de los estudiantes opinan que los nutrientes que son empleados en los cultivos sin tierra son cascarilla de huevo, compostaje, semillas de arroz y rocas; mientras que el 33\% opinan que solo es el compostaje.

- $83 \%$ de los estudiantes opinan que los cultivos hidropónicos germinan más fácil que un cultivo con tierra. 
- $87 \%$ de los estudiantes opinan que los cultivos hidropónicos no tienen plagas en comparación con los cultivos en tierra.

- Para el $37 \%$ de los estudiantes los aztecas fueron los primeros en usar la agricultura hidropónica eficientemente, mientras que el $23 \%$ opinan que fueron los mayas y el $20 \%$ opinan que ni los mayas, ni los aztecas, ni los hindúes, ni los egipcios.

La interpretación que dio el Grupo de Investigación fue que se pudo conocer que cada estudiante tuvo 5 preguntas correctas $(62,5)$ de las 8 que se realizaron a cada uno. Los estudiantes lograron encontrar características generales de un sistema de hidroponía.

05/14. Uso de las TIC para la investigación

- Registro de evidencias de cada proceso fotosintético de las plantas.

- Google Drive para la construcción del cuestionario.

05/15. Conclusiones y limitaciones. La principal dificultad se encuentra en el tiempo para trabajar y profundizar en las temáticas.

\section{5/R7. Lombricultivo y su efecto en los alimentos orgánicos.}

05/10. Introducción. Debido al manejo que se da de los residuos orgánicos en el Colegio nace la necesidad de aprovechar y convertir esos residuos en un beneficio, de ahí la idea de usar una alternativa como la lombricultura. El proyecto tiene como fin dar a conocer algunos aspectos básicos sobre el lombricultivo, teniendo en mente afianzar los conocimientos sobre el tema. Además de buscar las ventajas y los usos en el Colegio, específicamente.

05/I1. Instrumento. El objetivo del cuestionario es conocer la opinión de los estudiantes sobre la producción de alimentos orgánicos a través del compostaje producido en lombricultivo. Además, conocer la información que tienen los estudiantes sobre la lombricultura para aprovechar los desechos orgánicos y algunas opiniones sobre llevar estas iniciativas para usarse en el Colegio como un proyecto real. El resultado fue una encuesta con 7 preguntas con opción múltiple y única respuesta, en la cual se 
buscaba recoger las opiniones de los estudiantes sobre los lombricultivos y además diagnosticar el conocimiento de estos sobre estas iniciativas. En los encuentros realizados en el Grupo de Investigación, se vio la necesidad de saber qué tanto conocen los estudiantes del Colegio sobre los abonos orgánicos y qué pensaban sobre la implementación de este proyecto en el Colegio. El cuestionario fue elaborado con la participación de los estudiantes del Grupo de Investigación. Se realizó en Google Drive y se distribuyó a través de la Plataforma Virtual a los estudiantes de Educación Básica Secundaria del Colegio. Siendo una actividad complementaria de biología.

05/I2. Selección de la muestra. La población son los estudiantes de Educación Básica Secundaria del Colegio, al no ser muy grande la población, se dirigió el cuestionario a los 74 estudiantes de Educación Básica Secundaria. A pesar de esto, el cuestionario era voluntario lo que hizo que la participación de los estudiantes no fuera la muestra completa.

05/I3. Resultados. Se obtuvieron 28 respuestas completas para las 7 preguntas realizadas. Para analizar las respuestas que se obtuvieron se realizaron algunas sesiones en el Grupo de Investigación, estas tenían por objetivo discutir los resultados para lograr algunas conclusiones a partir de lo manifestado por los encuestados. Las preguntas más importantes para el análisis en el Grupo de Investigación fueron: ¿por qué es importante darles un uso adecuado a los desechos orgánicos?, ¿qué beneficios trae tener un lombricultivo en el Colegio?, ¿por qué es importante una sana alimentación?, y, ¿cree que es posible desde nuestros hogares dar el uso adecuado a los desechos orgánicos? Los resultados que arrojaron las encuestas fueron los siguientes:

- $86 \%$ de los estudiantes opinan que las lombrices se alimentan de residuos orgánicos.

- $54 \%$ de los estudiantes opinan que el mayor beneficio que tendría un lombricultivo en el Colegio es el aprovechar los residuos orgánicos de la cocina, mientras que el $43 \%$ opinan que el mayor beneficio es el obtener el abono orgánico.

- $50 \%$ de los estudiantes dicen que conocen los beneficios de un producto cultivado con abono orgánico, mientras que el $39 \%$ dicen no conocer estos beneficios.

- En una calificación de 1 a 5, el 36\% de los estudiantes opinan que el aprovechamiento de los residuos de la cocina para realizar abono orgánico a través del lombricultivo en el Colegio 
es de 3 , el $25 \%$ califican con 4 , el $21 \%$ califican con 5 y un $11 \%$ califican con 1.

- $57 \%$ de los estudiantes manifiestan que no comerían un alimento que fuera tratado con abono a base de lombrices, mientras que el $36 \%$ dicen que tal vez comerían de un alimento en estas condiciones.

- 78\% de los estudiantes dicen que el lombricultivo aportaría mucho en el Colegio, mientras que el $18 \%$ opinan que poco aportaría.

- 82\% de los estudiantes opinan que el lombricultivo aportaría a mejorar el ambiente ecológico en la granja.

Se realizó un análisis grupal de los resultados y el Grupo de Investigación pudo constatar que hay mucho desconocimiento del tema por parte de los encuestados. Además, se pudo conocer que los estudiantes han escuchado de los términos lombricultivo, manejo de residuos orgánicos, abono orgánico, entre otros, pero en sí no saben qué implica o qué tipo de abono se utiliza para fertilizarlo. Por lo tanto, debe realizarse una instrucción por parte del Grupo de Investigación a los demás integrantes de la institución.

05/14. Uso de las TIC para la investigación

- Encuesta: Google Drive.

- Video sobre el proyecto (producto): iMovie.

- Presentación (producto): prezi

05/15. Conclusiones y limitaciones. Es importante para el Colegio poner en funcionamiento un sitio de compostaje como el lombricultivo, para aprovechar los residuos orgánicos y con esto prolongar su vida útil en la utilización de abono orgánico. La mayor limitación es el tiempo destinado a la investigación, ya que no permitió la realización de las jornadas experimentales que se hubieran deseado.

\section{5/R8. "Welcome to Colombia"}

05/10. Introducción. El Proyecto de Investigación busca apoyar procesos educativos interculturales entre Colombia y Canadá. Para lo tanto, cada 
estudiante seleccionó un sitio turístico, con ayuda de sus padres, para realizar un video en inglés mostrando el sitio e invitando a los extranjeros a conocerlo. Para este producto fueron necesarias las siguientes actividades: realización del video, subida del video y generación del respectivo código QR, ubicación de los códigos en un mapa de Colombia, envío del mapa a estudiantes de Mississauga (Canadá) para que ellos interactuaran con el mapa. Trabajo con el objetivo de aportar a la cultura y sus procesos de multiconocimiento, por medio de un recorrido por diferentes sitios turísticos de Colombia.

05/11. Instrumento. Los objetivos con este cuestionario son: conocer las preferencias turísticas en Colombia, identificar los lugares turísticos más comunes de viaje, conocer las prioridades de los padres con respecto a los intercambios internacionales y saber la importancia del desarrollo de la lengua inglesa en los estudiantes, según sus padres. El cuestionario resultante está compuesto por 5 preguntas con opción múltiple y única respuesta. El cuestionario se construyó con ayuda de todos los miembros del Grupo de Investigación, ellos debían proponer preguntas las cuales se discutían en el grupo $y$, teniendo en cuenta los objetivos que se buscaban con el cuestionario, se aceptaban y se incluían. El cuestionario se construyó en Google Drive. El enlace para diligenciar el cuestionario se distribuyó por medio de la Plataforma Virtual del Colegio.

05/I2. Selección de la muestra. La muestra participante son los estudiantes de una Institución Educativa del Municipio de Chía, en total son 214 estudiantes y sus padres, distribuidos desde grado Primero a Décimo. La muestra, en principio, eran todos los estudiantes, pero al ser el cuestionario voluntario solo 38 personas respondieron.

05/I3. Resultados. Se obtuvieron 38 respuestas de las 5 preguntas realizadas. Sobre la preparación, el profesor titular de la investigación destaca que la etapa de campo fue crucial para el proyecto, ya que R4/10 "se obtuvo material importante para mostrar en Canadá y en la comunidad educativa (los videos)". Los resultados que arrojó esta encuesta fueron los siguientes:

- La mayoría de los estudiantes (71\%) opinan que el interculturalismo es conocer diversas culturas mundialmente de modo horizontal en el que ninguno está por encima del otro, el $13 \%$ dice que es conocer y participar en diferentes eventos de mi país y otro $13 \%$ dice que son acciones encaminadas a 
mantener el dominio de una determinada cultura, valores o pautas.

- Los estudiantes opinan en un $50 \%$ que el generar oportunidad de relacionarse con estudiantes extranjeros es la necesidad para un intercambio entre el Colegio y un Colegio de Canadá, mientras que un $37 \%$ opina que es para fortalecer el conocimiento de su idioma (inglés en este caso), por otro lado, un $13 \%$ dice que es necesario para fortalecer el conocimiento de los lugares turísticos de Canadá y ningún estudiante hizo alusión a que la necesidad fuera fortalecer el conocimiento de los lugares turísticos de Colombia.

05/I4. Uso de las TIC para la investigación

- Videos para promocionar el proyecto.

- Taller en línea sobre qué es un código QR y cómo se crea.

- Búsqueda de información - Internet.

- Video del lugar turístico.

- YouTube para alojar videos.

- Aplicación smartphone para crear los códigos QR.

- Herramientas para crear mapa de Colombia con los códigos QR.

- Skype para la presentación del proyecto en el Colegio canadiense.

05/I5. Conclusiones y limitaciones

- El mapa con los códigos QR funcionó como un aporte positivo en la muestra cultural de Colombia en un país extranjero. Además, se aprovechó que algunos estudiantes viajaron por Colombia para tener videos de diferentes sitios del país.

- El uso de nuevas herramientas TIC ayudan a establecer un contacto más cercano con la comunidad educativa.

\section{5/R9. La deforestación en Colombia}

05/I0. Introducción. Este Proyecto de Investigación busca determinar el conocimiento que tienen los estudiantes sobre las causas y consecuencias 
de la deforestación en Colombia, analizar las regiones más afectadas, conocer la percepción de los estudiantes sobre la problemática, conocer si en el Colegio hay una reflexión general entre los estudiantes de las consecuencias de la deforestación a nivel ambiental. Además, busca concientizar a los estudiantes del Colegio sobre esta problemática.

05/I1. Instrumento. Los objetivos del cuestionario son: conocer el concepto que tienen los estudiantes sobre la deforestación en Colombia, identificar las causas de la deforestación, analizar las regiones más afectadas por la deforestación en el país, dimensionar las consecuencias de la deforestación en Colombia, observar si en la institución hay reflexión sobre las consecuencias de la deforestación a nivel ambiental y conocer si los estudiantes entienden la diferencia entre reforestación y deforestación.

El cuestionario consta de 9 preguntas de selección múltiple con única respuesta, 4 con solo sí y no como posibles respuestas. En general, busca el diagnóstico del concepto de los estudiantes sobre la deforestación y específicamente la deforestación en Colombia. Basándose especialmente en analizar el impacto ambiental de la deforestación en Colombia y en el estudio de las regiones más afectadas este país. Además, teniendo como fundamento los objetivos que se buscaba evaluar el Grupo de Investigación, con una base conceptual, se realizaron en grupo las preguntas para realizar el diagnóstico (Ortiz, Buitrago, 2017). Se construyó en la página web e-encuesta.com y se distribuyó por el correo institucional.

05/I2. Selección de la muestra. Estudiantes de Segundo a Décimo grado del Colegio. Estudiantes de 8 a 15 años. En total son 170 estudiantes. Se seleccionaron 30 estudiantes, de manera aleatoria, a los que se les envió el cuestionario por correo electrónico.

05/I3. Resultados. Se obtuvo 30 respuestas de las 9 preguntas. Para el Grupo de Investigación, las preguntas que más ayudaron a orientar el análisis fueron: la pregunta que hace referencia a las consecuencias de la deforestación, para conocer la opinión de los estudiantes a nivel ambiental y la pregunta referente a conocer dónde se conoce el término deforestación, para conocer la información disponible en Centros Educativos. Los resultados que arrojaron las encuestas fueron los siguientes:

- $87 \%$ de los estudiantes dicen conocer las causas y las consecuencias de la deforestación.

- $55 \%$ de los estudiantes han escuchado sobre la deforestación 
por los medios de comunicación, el 29\% lo han hecho en el Colegio y el $16 \%$ lo han hecho en sus familias.

- $87 \%$ de los estudiantes consideran que la deforestación afecta a Colombia.

- $65 \%$ de los estudiantes opinan que la causa de la deforestación es el comercio de la industria maderera, el 19\% opinan que es el uso de los suelos para la ganadería y el $13 \%$ acusan que no saben o No Responden.

- 65\% de los estudiantes afirman que la deforestación afecta a Colombia en la pérdida del hábitat de los animales, mientras que un $19 \%$ dicen que en la reducción en las cuencas hidrográficas y el $16 \%$ opinan que no afecta a Colombia.

- $87 \%$ de los estudiantes consideran que es necesario analizar las consecuencias de la deforestación en Colombia en las instituciones educativas. 53\% opinan que la región amazónica es la que más ha sido afectada por la deforestación, el 27\% opinan que ha sido la región andina y un $20 \%$ dicen que la Orinoquía, ninguno opina que la región pacífica sea la más afectada.

- $71 \%$ de los estudiantes dicen conocer el concepto de reforestación.

- 42\% de los estudiantes manifestaron que la destrucción de bosques es el término que más se asemeja al concepto de reforestación, mientras que el 32\% indicó que el concepto más cercano era la plantación de árboles.

La interpretación que el Grupo de Investigación dio a estos resultados fue:

- Se hace necesario que en las instituciones educativas haya espacios de reflexión sobre la problemática ambiental de la deforestación, ya que a los estudiantes les falta información para dimensionar la deforestación en su país.

- 84\% de las personas acertaron en las principales causas de la deforestación en nuestro país, en la pregunta que se les realizó sobre este ítem.

- Los estudiantes que han escuchado sobre la deforestación, desean profundizar más sobre esta problemática.

- Existe una confusión en los estudiantes en el momento de usar el término reforestación, ya que a pesar de que los estudiantes en un $71 \%$ dicen conocer el término, solo un $42 \%$ acertaron en el momento de definirlo. 
05/14. Uso de las TIC para la investigación

- Creación, distribución, recopilación de resultados y visualización de resultados de encuestas: e-encuestas.com.

- Powtoon para la realización de historietas.

- Videos publicados en YouTube.

- Emaze para la exposición final del proyecto.

05/15. Conclusiones y limitaciones. Los estudiantes tienen un concepto sobre deforestación y, en su mayoría, lo han escuchado por los medios de comunicación. Además, los estudiantes ven necesario hacer un análisis sobre esta problemática ambiental en Colombia haciendo un énfasis en la región amazónica. La mayor limitación fue la falta de tiempo, a pesar de esto, los objetivos se cumplieron satisfactoriamente.

\section{5/R10. Visto y no visto}

05/10. Introducción. Estudio de la imagen como documento histórico es una necesidad para convertirse en una estrategia educativa. El interés del Proyecto de Investigación está centrado en dar uso a la imagen como un documento histórico, para fomentar el uso de este tipo de documentos y para conocer las dificultades que puedan ir apareciendo a la hora de su utilización. Actualmente, hay muy pocos historiadores que consultan los archivos fotográficos, comparado con los que consultan los depósitos de documentos manuscritos o impresos. En algunos casos las imágenes constituyen prácticamente el único testimonio existente de ciertas prácticas sociales como la caza, pero cuando se realizan estudios de épocas posteriores (épocas en las que ya existía la escritura) no se toman tan en serio las imágenes. Por lo tanto, esta investigación busca ver las imágenes no como limitación, sino como una fase de la investigación que se complementa con otros documentos históricos.

05/I1. Instrumento. Los objetivos del cuestionario son: opinión sobre las imágenes como una manera de entender un tema, preferencias de los estudiantes para la recopilación de información, opinión de los estudiantes de sobre el uso de imágenes como un recurso histórico, conocer si para el estudiante el contexto se debe tener en cuenta para analizar una imagen, intentar que los estudiantes deduzcan la trama de "Juego de tronos" de George R. R. Martin a partir de algunas imágenes, conocer la opinión y 
la sensación de los estudiantes con respecto a algunas imágenes y algunos sonidos. El cuestionario resultante consiste en 10 preguntas. 9 de las cuales son de opción múltiple con única respuesta y una pregunta abierta. Con respecto a la construcción, se mantuvo un registro de información que se quería obtener del tema, se estudiaron las opiniones de los estudiantes del Grupo de Investigación y se realizó una retroalimentación del cuestionario. Las preguntas fueron elaboradas por los integrantes del Grupo de Investigación, con base en la información recopilada y el conocimiento que fueron adquiriendo sobre la serie a lo largo del proceso investigativo. Teniendo en cuenta que se debía hacer un enfoque hacia el uso de imágenes como recursos históricos, se hizo necesario el uso de un grupo de respuestas para guiar a los estudiantes. El cuestionario se construyó en Google Drive.

05/12. Selección de la muestra. La población son los estudiantes de Educación Secundaria y Media Académica del Colegio. La muestra era la misma población de 109 estudiantes, ya que no era un grupo muy grande, pero dependía del apoyo de los estudiantes para responder el cuestionario, ya que se debía diligenciar de forma voluntaria.

05/I3. Resultados. Se obtuvieron 27 respuestas de las 10 preguntas que se realizaron. Para la preparación y el análisis previo se contó con información de la serie, conocimiento general del programa, interpretación de la imagen y contenido del tema. Además, se abrió un espacio de socialización sobre el conocimiento adquirido de la serie y sobre la temática. Se comenzó también con la elaboración de los productos finales y se realizó un juego de memoria sobre la seria. Finalmente, se realizó un barrido de páginas de Internet para conocer las opiniones de las personas sobre los aspectos fuertes y débiles del programa, junto con las observaciones y sugerencias que realizan. Los resultados que arrojaron las encuestas fueron los siguientes:

- 52\% de los estudiantes dicen que preferirían recopilar datos de la serie por medio de video, mientras que un $26 \%$ dicen que prefieren recopilarlo por medio de imágenes aisladas.

- 30\% de los estudiantes dijeron que analizarían las imágenes como recurso histórico como aspectos culturales, también el $30 \%$ dice que como datos y el $26 \%$ dice que como documentos. - $41 \%$ de los estudiantes dicen que el ambiente en el que se encuentran es el aspecto que más tendrían en cuenta para 
analizar una imagen, el $26 \%$ dice que por los personajes que aparecen y un 22\% dicen que por los hechos históricos.

- 37\% de los estudiantes dicen que pueden interpretar tiranía en las imágenes de Juego de tronos, el $30 \%$ interpretan violencia, el $19 \%$ dicen que incesto y el $15 \%$ dice que hay muchas mujeres.

- $52 \%$ de los estudiantes manifestaron que opinan que la Edad Media es el contexto histórico en el que fue desarrollada Juego de tronos, el 33 \% dicen que en la edad de la mitología nórdica y un $11 \%$ dicen que en la edad de oro.

- La mayoría de los estudiantes (59\%) identificaron en la imagen que se les presentó poder y liderazgo, mientras que el 33\% identificaron tiranía y ambición.

- De las imágenes de dos familias opuestas de la serie Juego de tronos, el 37\% de los estudiantes dicen que evocan bondad y arrogancia, el 30\% dicen que evocan hipocresía y envidia, el $22 \%$ humanidad y condescendencia, mientras que el $11 \%$ dicen que evocan petulantes y nobles.

- De las características que pueden sacar de las imágenes para entender el tema, los encuestados, en su mayoría (59\%), dicen que son el poder y la guerra, el 19\% dicen que intriga y drama, mientras que el $15 \%$ dicen que es el heroísmo y batalla, por otro lado, un 7\% dijo que el temor y fantasía.

- 37\% de los estudiantes dicen que la canción de entrada de la serie expresa gloria, el 33\% dicen que expresa valentía, el $19 \%$ dicen que expresa guerra, mientras que un $7 \%$ dicen que expresa orgullo.

La interpretación que dio el Grupo de Investigación sobre estos datos fue la siguiente:

- Los datos mostraron que las imágenes dan un mayor entendimiento de la serie y facilita el entendimiento del tema debido a que permiten mostrar lo que está ocurriendo o ciertas características de la serie.

- Las imágenes pueden ser usadas como recurso histórico ya que dan a conocer datos de los aspectos culturales que hay en la serie. La población también manifiesta que para interpretar una imagen es necesario conocer el contexto en el cual está. 
05/14. Uso de las TIC para la investigación

- Internet para la consulta de trabajos previos, para ver documentales y reportajes.

- Libros Digitales.

- Powtoon, YouTube, infogramas - desarrollo de productos del proyecto.

- Mapas conceptuales.

- Encuesta - Google Drive.

- Serie en línea.

\section{5/15. Conclusiones y limitaciones}

- Se buscó conocer a lo largo del proyecto qué tanto sabían los estudiantes sobre la saga "Juego de tronos", teniendo en cuenta gustos y preferencias de los encuestados respecto a la serie.

- También se buscó conocer cómo los estudiantes se relacionan mediante las imágenes, además de conocer cómo relacionan las imágenes con los sonidos. Dándole a las imágenes el mismo valor que un documento escrito.

- Complicaciones en la comunicación y organización por lo heterogéneo del grupo. Además, una de las principales limitaciones fue la falta de tiempo para las sesiones del grupo.

\section{Conclusiones}

Teniendo en cuenta la diversidad de temáticas desarrolladas en los Grupos de Investigación, se puede notar que los instrumentos que construyeron se pueden dividir en dos: encuestas diagnósticas sobre los conocimientos que poseen con respecto a un tema, destinadas al afloramiento, toma de conciencia y comunicación de los conocimientos previos, así como de errores, del "falso sentido común", de "agujeros conceptuales", etc., y cuestionarios para conocer diversas opiniones.

En cuanto a la manera de distribución del cuestionario, ya que la población/muestra participante está constituida por estudiantes del Colegio, los Grupos de Investigación escogieron la Plataforma del Colegio o el correo electrónico para distribución de cuestionario. 
Uno de los aspectos que más llamaron la atención por parte del observador fue la elección de las muestras para realizar el cuestionario. Los Grupos de Investigación usaron como muestra participante el grupo de estudiantes a los que el profesor encargado de la investigación les imparte clase. Por lo tanto, sí el profesor era de Educación Básica Primaria, la muestra participante eran los estudiantes de Educación Básica Primaria del Colegio, mientras que, si el profesor era de Educación Básica Secundaria, la muestra participante eran los estudiantes de Educación Básica Secundaria del Colegio.

En cuanto a los resultados, cabe destacar que no era necesaria una gran preparación o un análisis previo muy profundo, ya que, primero, la mayoría de las preguntas son cerradas, además, la herramienta que usaron daba como resultado gráficas de resumen y los resultados ya apilados (Google Drive). En la mayoría de los Proyectos de Investigación lo que buscaron fue que los estudiantes tuvieran el mayor conocimiento posible sobre el tema que estaban tratando para que en el análisis (usualmente por socialización) pudieran ser elementos importantes.

Uno de los aspectos más destacables en las respuestas que dieron los profesores durante la aplicación del cuestionario fue el hecho de que todos acusan falta de tiempo para desarrollar los proyectos o para la realización de las sesiones. A pesar de esto, dicen que los objetivos que se plantearon al inicio del proyecto fueron cumplidos.

Para finalizar, se puede resaltar que las TIC fueron fundamentales para los Proyectos de Investigación. Esto se debe a que permitieron a los estudiantes acceder a una gran cantidad de información, entre la que se encontraban los trabajos previos y muchos datos de los cuales pudieron basarse. Además, estas herramientas permitieron que el cuestionario fuera construido, distribuido, recopilado y analizado de una manera muy sencilla con herramientas como Google Drive o e-encuestas.com, lo que permitió que la recolección de información fuera eficiente. Siguiendo en la misma línea, las TIC también ayudaron a que se pudieran realizar múltiples productos en estas investigaciones de los cuales resaltan los juegos de los grupos de matemáticas y el mapa interactivo del grupo de inglés. También, les permitió a los profesores responsables por cada proyecto realizar un seguimiento y monitoreo constante y eficiente, por lo que hacer este seguimiento no les tomaba mucho tiempo y los dejaba libres para otras actividades. Finalmente, las TIC ayudaron a los Grupos 
de Investigación a dar a conocer los resultados o a intentar concientizar a la comunidad educativa del Colegio de una manera bastante sencilla.

\section{Referencias bibliográficas}

Aparicio, Ó. Y. (2015). Las TIC como herramienta cognitiva para la investigación escolar (Tesis doctoral, Universitat de Barcelona, Barcelona, España). Recuperado de http://hdl. handle.net/10803/369830

Aparicio, O.Y. (2018). Las TIC como herramientas cognitivas. Revista Interamericana de Investigación, Educación y Pedagogía, RIIEP, 11(1). https://orcid.org/0000-0003-35356288

Almeida, R. R., Santos, M. F., \& Porto, J. C. (2017). Lectura de textos ficcionales y el enfoque escolar de literatura: contribuciones para una Pedagogía de la Elección. Revista Interamericana de Educación, Pedagogía y Estudios Culturales, 9(1), 35-51. DOI: https:// doi.org/10.22490/25391887.1925

de la Calle, C. V., Malaver, M. O., Gallego, J. D. M., Rodríguez, M., Flórez, J. C., Henao, C. E. \& Saldaña, R. (2014). Aportes de los doctorados de educación en ciencia, tecnología y sociedad, desde la sistematización de sus investigaciones doctorales científicas y formativas, 2000-2010. Revista Interamericana de Investigación, Educación y Pedagogía, RIIEP, 7(1). DOI: https://doi.org/10.15332/s1657-107X.2014.0001.04

Konieczny, P. (2015). Lorenzo García Aretio: bases, mediaciones y futuro de la educación a distancia en la sociedad digital. Revista Interamericana de Investigación, Educación y Pedagogía, RIIEP, 8(1). DOI: https://doi.org/10.15332/s1657-107X.2015.0001.08

Ortiz, J. G., \& Buitrago, H. (2017). La evaluación en la tradición educativa colombiana. Instrumento de clasificación social. Revista Interamericana de Investigación, Educación y Pedagogía, RIIEP, 10(1), 145-171. DOI: https://doi.org/10.15332/s1657-107X.2148

Pérez, T. H. P. (2013). Aproximaciones al estado de la cuestión de la investigación en educación y derechos humanos. Revista Interamericana de Investigación, Educación y Pedagogía, RIIEP, 6(1). DOI: https://doi.org/10.15332/s1657-107X.2013.0001.05 